\title{
Evaluation of Oxidant Status and Lipid Profile In Vegetarians and Fish Eaters
}

\author{
Dr. Nalla. Viswabharathi ${ }^{*}$, Dr.S.R.Manjula ${ }^{2}$, K.Siddhartha ${ }^{3}$. \\ ${ }^{I}$ Assistant Professor, Department of Biochemistry, Siddhartha Medical College, NTRUHS, Vijayawada, India. \\ 2 Assistant Professor, Department of Physiology, SMC,NTRUHS, Vijayawada, India. \\ ${ }^{3}$ Seventh semester medical student, SMC, NTRUHS, Vijayawada, India. \\ Correspondence author: Postal Address: Dr. N. Viswabharathi
}

\begin{abstract}
Atherosclerosis is the leading cause of death and disability in the developed world. Habitual consumption of moderate amount of fish was associated with reduced mortality from coronary heart disease. It was also acclaimed that vegetarians in general will have low serum cholesterol and a better anti oxidant status than fish eaters. In view of this a comparative study of oxidant status and lipid profile was done in vegetarians and fish eaters. This study enrolled 50 vegetarians and 50 fish eaters of age 35-55 years.

Statistical Analysis by the students "T" Test or Mann Whitney "U" Test was applied depending upon the nature of the data. Pearson's correlation co efficiencies were used to compare the correlations. It showed significant difference in HDL-C levels among fish eaters when compared to vegetarians; this study showed no significant difference in the lipid peroxide levels and the other lipoproteins. The correlation of malondialdehyde (MDA) with HDL-C was negative in the vegetarians and weakly positive in the fish eaters. $L D L-C$ showed a positive correlation with MDA in both the groups. Triglycerides showed a positive correlation with MDA and correlation was strong in fish eaters.
\end{abstract}

Key words: Atherosclerosis, coronary artery disease, Lipid profile, Oxidants, Antioxidants, Nutrients in fish.

\section{Introduction}

Atherosclerotic disease is the leading cause of mortality in developed countries with Coronary Artery Disease (CAD) being the number one killer of both men and women. Heart Disease is preventable; researchers have been trying for years to find the best ways to reduce the prevalence of it in our society. Besides lifestyle changes, research is in progress for both nutritional and drug treatments. One of these nutrient studies is Omega3 fatty acids. These long chain polyunsaturated fatty acids are found in high concentration in fish and fish oil and are thought to have preventive effects on heart disease. Studies are therefore done on Omega-3 fatty acid supplements, fish and fish oil and their possible relations to heart disease.

In today's world, most deaths are attributable to non communicable diseases (32 million) and just over half of these 16.7 millions are as a result of Cardio Vascular Disease (CVD); more than one third of these deaths occur in middle aged adults. In developed countries, heart diseases and stroke are first and second leading cause of death for adult men and women. Coronary Heart Disease (CHD) is a worldwide disease. Mortality rates vary widely in different parts of the world. The Highest coronary mortality is seen at present in the North Europe and in English speaking countries (Scotland, Northern Ireland, and Finland) on the other hand the rates in southern Europe are much lower and those in Japan, although a rich industrialized country, are extremely low. In India, an estimated 1.5 million people died due to CVD every year; by 2030 this loss is expected to rise to 17.9 million years. The prevalence of IHD among adults based on clinical and ECG criteria was estimated as $96.7 \%$ population in the urban and $27.1 \%$ in rural areas .[1]

The present mortality rates are the consequences of previous exposure to behavioral risk factors such as inappropriate nutrition (fast food habits), insufficient physical activity leading to overweight, central obesity and dyslipidemia. It is now well established fact that a persistently high cholesterol level can almost certainly precipitate a cardiac event such as Coronary Heart Disease. It is nearly 3 decades since it became clear that elevation of serum cholesterol was one of the factors which carried an increased risk for the development of myocardial infarction. It is the level of low density lipoprotein (LDL) that is most directly associated with CHD; while very low density lipoprotein (VLDL) has also been shown to be associated with peripheral vascular disease (eg: Intermittent Claudication) than with CHD. High density lipoprotein (HDL) is protective against the development of CHD- the higher its mean level in a group of individuals, the lower the incidence of infarction in that group. HDL should be more than 40 milligrams per deciliter. Today, there is a vast body of evidence showing a triangular relationship between habitual diet, blood cholesterol, lipoprotein levels and coronary heart diseases. 
It is well established that free radical damage contributes to the etiology of cardiovascular diseases as well as other diseases like Carcinoma, cataract, diabetes etc; there is growing interest in the possible role of free radicals in the development of Coronary Heart Disease. Free radicals can cause oxidation of low density lipoproteins (LDL). Oxidized LDL has been demonstrated to accelerate endothelial damage, macrophage recruitment and increased uptake of LDL-C by foam cells which gradually develop into fatty streaks.

Lipid peroxidation has been established as a major mechanism of cellular injury in many biological systems of plant and animal origin. The mechanism involves a process whereby unsaturated lipids were oxidized to form additional radical species as well as toxic by-products that were harmful to the host system. Polyunsaturated lipids were especially susceptible to this type of damage when in an oxidizing environment and they react to form lipid peroxides. Lipid peroxides were themselves unstable, and undergo aditional decomposition to form a complex series of compounds including reactive carbonyl compounds. Polyunsaturated fatty acid peroxides further react to form malonaldehyde (MDA). Lipid peroxidation [2] and consequent degradation products such as malondialdehyde (-CHO-CH2-CHO-) were seen in biological fluids there estimation in serum was often employed to assess the oxidant status. Oxidant means the electron acceptor in an oxidation reduction (redox) reaction. The present study aims to estimate the serum malondialdehyde (MDA) by Thio Barbiburic Acid (TBA) calorimetric assay. Free radical theory [3] and its importance to disease and aging was initially articulated in 1954 by Denham and Harman . [4]

The following were the free radicals in the living cells - Superoxide anion $\left(\mathrm{O}_{2}^{-}\right)$the partially reduced hydrogen peroxide $\left(\mathrm{H}_{2} \mathrm{O}_{2}\right)$ and hydroxyl free radical $\left(\mathrm{OH}^{*}\right)$. Of these intermediaries in reduction of $\mathrm{O}_{2}$ to water, the hydroxyl radical is undoubtedly the most dangerous free radical as it is involved in reactors such as lipid per oxidation and generation of other toxic radicals. Hydrogen peroxide itself is not a free radical but was converted by the Fenton or Haber-Weiss reactions to the hydroxyl radical in the presence of $\mathrm{Fe}^{2}+$ or calcium prevalent in cells. Reactive oxygen species (ROS) mediated oxidative damage macromolecules namely lipids, proteins and DNA had been implicated in the pathogenecity of major diseases such as cancer,[5] rheumatoid arthritis, post ischemic reperfusion injury, myocardial infarction, cardiovascular disease etc. [6] The damage produced by ROS were prevented by anti-oxidants and Scavenging enzymes. Preventive anti-oxidants inhibit the initial production of free radicals. They are catalase, glutathione peroxidase, diethyl triamine penta acetate (DTPA), and ethylene diamine tetra acetate (EDTA). Chain Breaking anti - oxidants like Superoxide Dismutase, Uric acid and Vitamin E (Alpha Tocopherol, Betacarotene) and other anti - oxidants were Vitamin C, ceruloplasmin, caffeine, uricacid, and vitamin A.

Vegetarian diets are associated with a number of health benefits: lower risk of death from heart disease, lower low density lipoprotein (LDL) cholesterol levels, lower blood pressure, lower rates of type 2 diabetes, lower body mass index, and lower rates of cancers. [7] The primary dietary factor that likely confers these benefits is the increased consumption of whole plant foods (fruits, vegetables, whole grains, seeds, nuts, beans) and associated beneficial nutrients V fiber, antioxidants, vitamins, minerals and phytochemicals. [8]

Fish is an excellent source of complete proteins, Lipids, phosphorous, Iron, and other minerals, some are high in vitamin A and fish is a rich source of Omega-3 fatty acids. Sea fish contains Iodine; liver of white fish contains vitamins A, D and E. Eating fish three times a week has been associated with a significant decrease in the rate of heart disease; this became apparent when Scientists noted that coronary artery disease- a leading cause of death in North America - was almost nonexistent among the indigenous people of Greenland, Japanese fisherman, and first nation of the Pacific Northwest. The one factor that these three groups had in common was a diet that relied heavily on fish for proteins. When researchers looked at the effects of diets in other population they found that men who ate fish regularly two or three times a week was much less likely to suffer Heart attacks than others. In view of the present scenario, this work was taken up to study the oxidant status and lipid profile in vegetarians and fish eaters.

\section{Materials and Methods}

This study was conducted on 100 subjects out of them 50 were vegetarians, 50 were predominantly fish eating people in the age group of 35-55 yrs. This study was approved by the Institutional ethics committee. Written informed consent was taken from the patients in local language. This was an observational, retrospective, cross sectional and comparative study. Age, height, weight and blood pressure were recorded and food habits of the subjects were obtained using a questionnaire 


\subsection{Inclusion Criteria:}

2.1.1. Vegetarians: Subjects consuming purely lacto- vegetarian diet.

2.1.2. Fish Eaters: Subjects consuming 4-6 fish dishes per week (4-6 fish meals/week) along with vegetables and occasionally other meals (approximately quality was calculated).

\subsection{Exclusion Criteria:} from the study.

For both groups, subjects who were smokers, alcoholics, diabetics or hypertensive's were excluded

2.3. Collection of Blood Samples: $5 \mathrm{ml}$ of heparinized venous blood was collected after an overnight fast of 12 hrs and the following parameters were estimated in the plasma with in $2 \mathrm{hrs}$ of sample collection. Serum was separated and

2.3.1. Estimation of total cholesterol by CHOD-PHOD/phospho tungstate method of Allianc [9],

2.3.2. Estimation of High Density Lipoprotein (HDL) cholesterol by CHOD - POD phosphotungstate method by Burstein [10],

2.3.3. Estimation of triglycerides by glycero phosphate oxidase method of jacobe [11],

2.3.4. Estimation of LDL by fride wald equation [12]:

LDL cholesterol $=$ cholesterol $-($ HDL + VLDL $)$. and

2.3.5. Estimation of MDA levels (Malondialdehyde) by Thiobarbituric Acid (TBA) reaction for MDA (Mahalouz et al 1978) was used. Principle: Under the acidic and the treating conditions of the reaction the lipid peroxides breakdown to form Malondialdehyde which complexes with Thiobarbitutic acid (TBA). The resulting MDA-TBA chromogen is measured at 530nm distilled against distilled water, in a spectrophotometer. [13]

2.4. Body Mass Index (B.M.I) [14] was calculated in both men and women as follows:

$\mathrm{BMI}=$ Weight in $\mathrm{Kg} /$ Height in (meters) ${ }^{2}$

2.5. Atherogenic Index: were indicated by various risk factors like T.C/HDL-C, LDL/HDL, TC/HDL $\times$ MDA and $\mathrm{LDL} / \mathrm{HDL} \times \mathrm{MDA}$.

2.6. Statistical Analysis: Statistical Analysis was done by the students "T" Test or Mann Whitney "U" Test was applied depending upon the nature of the data. Pearson's correlation co efficiencies ( $r$ value) were used to compare the correlations.

\section{Results}

This study was initiated in August 2012 and completed in July 2013 at Government General Hospital and Siddhartha medical college, Vijayawada, Andhra Pradesh, India; and samples of fish eaters were collected from Machilipatnam (sea shore). Anthropometric data of the study groups (table no. 1) were comparable in age and BMI; all the subjects were normotensive.

TABLE: 1 ANTHROPOMETRIC DATA

\begin{tabular}{|l|l|l|l|}
\hline Sl.No & PARAMETERS & VEGETARIANS $(\mathrm{n}=50)$ & FISH EATERS $(\mathrm{n}=50)$ \\
\hline 1 & Age(Yrs) & $35.12 \pm 12.05$ & $47.76 \pm 4.33$ \\
\hline 2 & BMI & $22.23 \pm 2.89$ & $22.04 \pm 3.02$ \\
\hline 3 & Systolic BP(mm of hg) & $108.2 \pm 7.28$ & $99 \pm 15.10$ \\
\hline 4 & Diastolic BP (mm of hg) & $80 \pm 8$ & $72.2 \pm 5.11$ \\
\hline
\end{tabular}

$n=$ No of subjects, Values are mean \pm standard deviation, BMI=body mass index.

Plasma lipid peroxides and Lipid profile in Vegetarians and Fish Eaters were shown in table no 2: 
TABLE NO 2: DATA SHOWING LIPID PEROXIDE AND LIPID PROFILE

\begin{tabular}{|l|l|l|l|}
\hline Sl.No & PARAMETERS & VEGETARIANS $(\mathrm{n}=50)$ & FISH EATERS $(\mathrm{n}=50)$ \\
\hline 1 & MDA $(\mathrm{mmols} / \mathrm{L})$ & $202 \pm 36.6$ & $210 \pm 31.41$ \\
\hline 2 & Total Cholesterol $(\mathrm{mg} / \mathrm{dl})$ & $182.5 \pm 19.32$ & $188.56 \pm 25.01$ \\
\hline 3 & HDL $-\mathrm{C}(\mathrm{mg} / \mathrm{dl})$ & $44.3 \pm 6.94$ & $60.84 \pm 16.69$ \\
\hline 4 & LDL-C $(\mathrm{mg} / \mathrm{dl})$ & $108.46 \pm 20.48$ & $102.78 \pm 15.44$ \\
\hline 5 & VLDL-C $(\mathrm{mg} / \mathrm{dl})$ & $27.7 \pm 10.84$ & $24.35 \pm 7.52$ \\
\hline 6 & Triglycerides $(\mathrm{mg} / \mathrm{dl})$ & $92.6 \pm 43.39$ & $121.84 \pm 33.19$ \\
\hline
\end{tabular}

$n=$ No of subjects, Values are mean \pm standard deviation, $M D A=$ malondialdehyde, $H D L-C=$ high density lipoprotein, $L D L-C=$ low density lipoprotein, $V L D L-C=$ very low density lipoprotein.

The mean content of Plasma MDA was $202 \pm 63.62$ in vegetarians and $210 \mathrm{mmol} / \mathrm{L} \pm 31.41$ in fish eaters the difference between the two groups was not significant. The mean of HDL cholesterol and Triglycerides were increased in case of fish eaters. Other lipid parameters cholesterol, LDL-C, VLDL-C did not showed any significant difference between vegetarians and fish eaters; the values in both the groups were well within the normal limits.

The $r$ values for the correlation of MDA with lipid parameters were given in table no 3.

TABLE NO: 3 CORRELATION OF MDA WITH LIPID PARAMETERS “r” VALUES

\begin{tabular}{|l|l|l|l|}
\hline S.No. & PARAMETERS & VEGETARIANS $(\mathrm{n}=50)$ & FISH EATERS $(\mathrm{n}=50)$ \\
\hline 1 & Total Cholesterol $(\mathrm{mg} / \mathrm{dl})$ & 0.373 & 0.088 \\
\hline 2 & HDL-C $(\mathrm{mg} / \mathrm{dl}$ & -0.507 & +0.884 \\
\hline 3 & LDL-C $(\mathrm{mg} / \mathrm{dl})$ & 0.128 & 0.191 \\
\hline 4 & VLDL-C $(\mathrm{mg} / \mathrm{dl})$ & 0.229 & 0.656 \\
\hline 5 & Triglycerides $(\mathrm{mg} / \mathrm{dl})$ & 0.034 & 0.592 \\
$n=$ number & of subjects, HDL-C $=$ high density lipoprotein, LDL-C $=$ low density lipoprotein, \\
VLDL-C $=$ very low density lipoprotein.
\end{tabular}

The correlation of MDA with HDL-C was negative in the vegetarians $(\mathrm{r}=-0.507)$ and weakly positive in the fish eaters $(+0.884)$. LDL-C showed a positive correlation with MDA in both the groups. Triglycerides showed a positive correlation with MDA and correlation was stronger in Fish eaters $(r=0.592$ Vs 0.034$)$.

Atherogenic index was indicated by various risk factors were shown in table no 4.

\section{TABLE NO 4: ATHEROGENIC INDEX}

\begin{tabular}{|l|l|l|l|}
\hline Sl.No & PARAMETERS & VEGETARIANS $(\mathrm{n}=50)$ & FISH EATERS $(\mathrm{n}=50)$ \\
\hline 1 & T. CLHDL-C & $4.11 \pm 2.7$ & $2.00 \pm 1.5$ \\
\hline 2 & LDL/HDL & $2.44 \pm 3.33$ & $1.68 \pm 1.08$ \\
\hline 3 & TC/HDL X MDA & $832.16 \pm 1.10$ & $420.55 \pm 20.6$ \\
\hline 4 & LDL/HDL X MDA & $494.55 \pm 13.33$ & $354.64 \pm 28.7$ \\
\hline
\end{tabular}

$n=$ Number of Subjects, Values are Mean \pm standard deviation, T.C= total cholesterol, $M D A=$ Malondialdehyde.

TC/HDL-C ratio was higher in vegetarians when compared to fish eaters. The ratio of LDL/HDL was also a little higher in vegetarians in comparison to fish eaters. Since increased lipid per oxidation was also a risk factor for CHD; MDA values were multiplied by risk ratios to obtain a new index which serves as a better predictor of CHD however they were not statistically significant. The relationship between cholesterol and Atherosclerotic coronary disease was not linear, if a risk ratio of 1.0 is assigned at the cholesterol value of $200 \mathrm{mg} / \mathrm{dl}(5.18 \mathrm{~m}$ $\mathrm{mol} / \mathrm{L})$; the risk ratio increases to 2.0 at $250 \mathrm{mg} / \mathrm{dl}(6.48 \mathrm{~m} \mathrm{~mol} / \mathrm{L}) ;$ and 4.0 at $300 \mathrm{mg} / \mathrm{dl}(7.77 \mathrm{~m} \mathrm{~mol} / \mathrm{L}) 30$. 
Total Cholesterol by HDL-C ratio more than 3.5 was dangerous. Similarly, LDL-HDL ratio more than 3.5 was also deleterious.

\section{Discussion}

The $\omega-3$ (Omega) Poly Unsaturated Fatty Acid (PUFA) had a unique physiological effect that decrease the risk of heart disease: they decrease platelet aggregation and were thought to act by tromboxane $\mathrm{A}_{3}$, which was only weakly proaggregating, and prostaglandin $\mathrm{I}_{3}$ (PGI), which was strongly antiaggregation would be shifted towards a more antiaggregating condition. The $\omega_{-3}$ (Omega) Poly Unsaturated Fatty Acid (PUFAs) also acts by simply inhibiting the conversion of arachidonic acid to $\mathrm{TAX}_{2}$. Epidemiological studies on Greenland Eskimos had shown a correlation between low incidence of CHD and high consumption of fish products. [15] Dietary fiber played an important role in heart diseases by affecting atherogenesis. Habitual consumption of moderate amount of fish was associated with reduced mortality from CHD and was attributed to the Omega-3 fatty acids present in them; Bang \& Dyeberg [16] emphasized the importance of Omega-3 fatty acids in the prevention of myocardial infarction because of its anti thrombin and hypocholesteremic effect. On the other hand, vegetarians had a greater intake of omega6 series of fatty acids in their diet; differences in blood lipids between the vegetarians [17] and omnivorous had been well studied.

This study investigated the effect of moderate amount of fish in regular diet and vegetarians on the oxidant status and lipid profile. The results of this study indicated little or no significant difference in the lipid peroxide levels of vegetarians and fish eaters. This finding was in agreement with the report Higdon Et al [18] who studied in vivo oxidation by measuring plasma MDA and PGF2 - Isoprotanes. Bhattacharya Et al [19] had reported increased lipid per oxidation in hyper lipidemia. Ohara Et al [20] had reported increased superoxide anion production in hypercholesterolemia. Meydane Et al [21] and Piche [22] Et al had reported increased concentration of plasma MDA in humans given supplements of fish oil. The results of this present study gave credence to the argument that fish diet may not lead to increased oxidation in vivo. Some studies showed that long term pure vegetarians had very low levels of oxidants and cholesterol. [23]

Elevated levels of plasma Triglycerides (TG) had long been associated with the risk of Coronary Heart Disease (CHD); in this present study $46 \%$ of vegetarians and $60 \%$ of the fish eaters showed Triglycerides (TG) levels below the cutoff point $150 \mathrm{mg} / \mathrm{dl}$. Lack of significant difference in the serum Triglycerides (TG) of the study groups was consistent with the literature finding which showed that plasma Triglycerides (TG) were not affected by alterations in the saturated, monounsaturated and n-6 Poly Unsaturated Fatty Acid (PUFA). High Density Lipoprotein (HDL)-C had an inverse relation to the risk of Coronary Heart Disease (CHD). Person with below $35 \mathrm{mg} / \mathrm{dl}$ had a Coronary Heart Disease (CHD) incidence rate of more than 8 times compared to the persons with High Density Lipoprotein (HDL) levels greater than $65 \mathrm{mg} / \mathrm{dl}$ or above. In this study significant difference was observed between the fish eaters and vegetarians, in $22 \%$ of vegetarians HDL-C levels were less than $50 \mathrm{mg} / \mathrm{dl}$ and $30 \%$ of vegetarians had HDL-C levels more than $50 \mathrm{mg} / \mathrm{dl}$. Interestingly in this study a negative correlation of HDL-C with MDA was seen only in Vegetarians. A recent analysis reported the overall mean antioxidant content of plant foods to be 11.57 mmolI100 gj1. Compare this to the mean antioxidant content of animal foods $\mathrm{V}$ a minute $0.18 \mathrm{mmolI} 100 \mathrm{gj} 1$. [24]

Bryant Stamford, Director of the Health and Wellness centre at University of Louisville, says recent research provides modest support for fish oil. It must lower Blood pressure but only slightly 3-5 points. It might help lower cholesterol and Triglycerides but not to a greater extent. It might reduce clot formation, help keep cholesterol from being deposited in artery walls- the first step in atherosclerosis. But these effects have yet to be established. The non significant differences in this study group might be due to a small sample size so had to be correlated with a bigger sample. However the beneficial effects of fish enriched diet seems contradictory due to susceptibility of the Poly Unsaturated Fatty Acid in them to oxidation; Unlike in other populations, vegetarianism offers little protection against heart disease among Indians because Indian vegetarians tend to avoid fish and eat lot of saturated dairy fat, trans fats and high glycemic carbohydrates. [25]

\section{Conclusions}

The best proven way to prevent the onset of heart diseases is through life style modification. The risk of developing heart disease can be prevented in many ways. It could be through the change of habits in their own lifestyles. Exercising regularly, eating foods low in fat and salt, no smoking and deleting stress from our lives were some of the primary ways of keeping a healthy heart. However, eating fish is a healthy thing to do, regardless of whether you are getting a hefty dose of Omega -3 fatty acids. Recent research suggest that eating fish helps to prevent heart attacks, possibly because if you were eating fish you were not eating red meat, which was high in saturated fat. As with many of the illnesses that plague our society, several drugs and treatments 
have been discovered in order to address the problem once it is already implanted in a person. Some of these treatments are drugs that help to lower the cholesterol level in the blood while others are dietary management that naturally help to reduce the risk of getting heart diseases.

\section{References}

[1]. K. Park: Park's text book of preventive and social medicine. 2009 chapter 6: $317-322$.

[2]. Ester Banner H, Chesseman KH, 1990 Determination of Aldehyde lipid per oxidation products, malondialdehyde, and forehydroxy nonal methods in enzymology, Academic press, 186, 407-21

[3]. Harman D, (1994) Free radical theory of aging increasing the functional life span. Ann NY Acad Sci. 717 para $1-15$.

[4]. Denham Harman (1956): Aging a Theory based on free radical and radiation Chemistry, Journal of gerontology. 11 (3)Pg. $298-3007$.

[5]. Mohammad A.(2002); Oxidative stress and experimental carcinogenesis. Ind.J.Exp.Biol.40:p656-667

[6]. Wiseman H and HalliwellB (1996); Damage to DNA by reactive oxygen species; Role of inflammatory diseases and progression to Cancer.Biochem.J.313:P17-29

[7]. Craig WJ, Mangels AR. American Dietetic Association. Position of the American Dietetic Association: vegetarian diets. J. Am. Diet. Assoc. 2009; 109(7):1266Y82.

[8]. FUHRMAN, J. and D.M. FERRERI. Fueling the vegetarian (vegan) athlete. Curr. Sports Med. Rep., Vol. 9, No. 4, pp. 233Y241, 2010.

[9]. Allain C.C. Clinichem 20, 470 (1974).

[10]. Bustein, M. etal (1970) J. lipid res. 17:583

[11]. Jacobe, N.J., Van Demark, P.J. (1960) Arch Biochem. Biophys. 88,250

[12]. Tietz fundamentals of clinical chemistry sixth Edition: Edited by carl A. Burtis, Edward R. Ashwood David E. Bruns Chapter 23: p 424.

[13]. D'souza D, Babu G S, Shetty SR, Balan P. Estimation of serum malondialdehyde in potentially malignant disorders and postantioxidant treated patients: A biochemical study. Contemp Clin Dent 2012;3: 448-51

[14]. Lippin cott's illustrated reviews $3^{\text {rd }}$ Edition Chap. 26 Page 347.

[15]. Bang HO Dyberg J. (1972) Plasma Lipids and Lipo proteins in Green lands West Coastal 192, 85-94.

[16]. Bang HO Dyberg J. (1972) Plasma Lipids and Lipo proteins in Green lands West Coastal 200, 69-73

[17]. Sack FM, Wood PG, Kosh EH 1984, Stability of BP in Vegetarians receiving Dietary protein supplement HDN 6199 -201

[18]. Higdon J.V.Lice J.Morrow, JD,Ames BN Wander RC(2000) Supplimentation of postmenopausal woman with fish oil rich in EPA and DHA is not associated with grater in vivo lipid per oxidation compared with oils rich in oleate and linoleate as assessed by plasma Malondialdehyde and F2 -Isoprostanes:A.M.J.Clin Nutr 72,714-22.

[19]. Bhatta Charya J.Sri vastav DK(1993) Serum malondialdehyde in relation to Lipademic status and Atherogenic index :Indian jour of Clinical Bio 8:12-15.

[20]. OHARA Y. Peterson TE Harrison D G (1993) Hyper cholesterolemia increases endothelial superoxide anion production J CLIN.Invest91.2546-51.

[21]. Meydani M . Evans W J Handelruan G (1993) Protective effect of vitamin E on exercise induced oxidative damage in young and older adults AMJ Physiol.264: $992-8$

[22]. Piche LA : Draper HH cole PD (1988) malondialdehyde excretion by subject consuming Cod liver oil Vs a concentration of N3 fatty acids: Lipids 23:370-1

[23]. Mi Kyung Kim, ${ }^{1}$ Sang Woon Cho, ${ }^{1}$ and Yoo Kyoung Park . Long-term vegetarians have low oxidative stress, body fat, and cholesterol levels Nutr Res Pract. 2012 April; 6(2): 155-161.

[24]. Carlsen MH, Halvorsen BL, Holte K, et al. The total antioxidant content of more than 3100 foods, beverages, spices, herbs and supplements used world wide. Nutrition J. 2010; 9(3):1475Y2891.

[25]. Enas A. Enas, How to beat the heart disease among south Asians. 2008;1.3: 30-33. 